\title{
COHERENCIA Y VIGENCIA DE LA VIDA Y OBRA DEL SOCIÓLOGO ORLANDO FALS BORDA ${ }^{12}$
}

Coherence and validity of the life and work of the sociologist orlando Fals borda

\section{SUÁREZ FERNÁNDEZ, Normando José3}

Resumen: El autor desarrolla una reflexión sobre el carácter de la obra del gran sociólogo Orlando Fals Borda y de su compromiso para con los grupos y comunidades sociales más vulnerables. Las evidencias de la construcción, desarrollo, mejoramiento y afinamiento del método participativo están consignadas en sus escritos. La Investigación Acción Participativa es una contribución inestimable para la sociología de la acción. La publicación de Campesinos de los Andes y otros escritos antológicos por la Universidad Nacional de Colombia contribuyen para avanzar en la construcción de un paradigma holístico alterno. Esto es, de um paradigma basado en la investigación acción participante desde una perspectiva interdisciplinaria que trasciende a los enfoques positivistas, funcionales y mecánicos, aportando respuestas a las situaciones críticas de colombianas y colombianos.

Palabras-claves: Fals Borda. Sociologia colombiana. Método de investigación acción participativa. Antología.

\begin{abstract}
The author develops a reflection on the character of the the great sociologist Orlando Falz Borda studies and his commitment to the most vulnerable social groups and communities. Some evidences of the construction, development, improvement and refinement of the participatory method are recorded in their writings. Participatory Action Research is an invaluable contribution to the sociology of action. The publication of "Campesinos de los Andes" and other anthological writings by the National University of Colombia contribute to advance in the construction of an alternative holistic paradigm. That is, a paradigm based on participatory action research from an interdisciplinary perspective that transcends positivist, functional and mechanical approaches, providing a response to the critical situations of Colombians.
\end{abstract}

Keywords: Fals Borda. Colombian sociology. Participatory research method. Anthology.

\footnotetext{
1 Prólogo del libro: Orlando Fals Borda. Campesinos de los Andes y otros escritos antológicos. Edit. UNAL. 2017.

${ }^{2}$ Texto selecionado para este dossiê da REALIS

${ }^{3}$ Sociólogo, docente del departamento de Sociología de la Universidad Nacional de Colombia. E-mail: sunorma1@hotmail.com
} 
El compromiso ${ }^{4}$ de Fals Borda con los grupos y comunidades sociales más vulnerables tiene su origen en la orientación religiosa presbiteriana ${ }^{5}$, su paso por el colegio Americano de Barranquilla, la formación que le impartió su progenitora, maestra y líder de las campañas en el campo de la educación y salud María Borda Angulo, la influencia del pastor Richard Shaull ${ }^{6}$ con un enfoque social a la misión de la Iglesia y una clara orientación a la teología de la liberación, así como la incidencia de la Universidad Presbiteriana Debuque, Iowa, Estados Unidos, donde estudió Literatura Inglesa e Historia a partir de 1943. Como presidente del Centro Juvenil Presbiteriano, en la capital del departamento del Atlántico, comenzó a explorar el ecumenismo, compartiendo actividades sociales y artísticas con Alejandro Obregón y Álvaro Cepeda Zamudio, que luego formaron parte del Grupo de Barranquilla. Previo a su tránsito como director del coro de la primera iglesia presbiteriana de Bogotá compone "Mensaje a Colombia"7 en respuesta al impacto que le causó el asesinato de Jorge Eliécer Gaitán y los acontecimientos que se desataron después del 9 de abril de 1948, que marcaron el inicio de un nuevo ciclo de la violencia política en Colombia, hecho que desde entonces lo interpeló y lo llevó a estudiar con el sacerdote católico monseñor Germán Guzmán Campo y el librepensador Eduardo Umaña Luna. Ese espíritu ecuménico lo acentuó, después, al fundar la Facultad de Sociología en la Universidad Nacional de Colombia con el sacerdote católico y sociólogo Camilo Torres Restrepo compartiendo en su primera etapa su planteamiento teológico a través del compromiso del amor eficaz de los cristianos y traducido en un compromiso con la transformación de la realidad de los grupos más vulnerables de la sociedad.

A los 25 años, el compromiso de Fals Borda trascendió del plano confesional al de carácter más secular y de ética social, para sumergirse como sociólogo autodidacta a convivir con las familias de la vereda de Saucío, entre 1949 y 1951, con el propósito altruista de "analizar los resultados y formar con ellos un estudio que permitiera captar

\footnotetext{
${ }^{4}$ Cataño, Gonzalo, Orlando Fals Borda, sociólogo del compromiso. Revista de Economía Institucional 10, N. 19 (2008), pp. 79-98.

${ }^{5}$ Castillo Cárdenas, Gonzalo y Pérez Benavides Isay, La influencia religiosa en la conciencia social de Orlando Fals Borda (Barranquilla: Corporación Universitaria Reformada, septiembre de 2010).

6 Ibid. p. 44.

7 Ibid., p. 46
} 
los problemas de los campesinos como un conjunto integrado. Pero aun en el caso de que hubiera fracasado en ambos intentos, pensé que el esfuerzo valía la pena hacerlo"8 muy a pesar de no contar con apoyo financiero directo 9.71 Simultáneamente, al desempeñarse como secretario personal bilingüe, español-inglés, del director de la represa del Sisga en construcción por la firma americana Winston Brothers Company para la Caja Agraria, asumió de manera personal, lo que hoy se denomina "responsabilidad social empresarial" con la mano de obra campesina de la vereda de Saucío.

Su solidaridad con este vecindario se manifestó con la fundación y organización de la acción comunal, en 1958, para construir la escuela, el acueducto veredal y la tienda cooperativa hasta bien entrada la década del sesenta.

El compromiso de Fals Borda se fue acentuando y cualificando, mucho más, en su paso y retiro de la Universidad Nacional de Colombia y se fue perfilando con el descubrimiento del método de la Investigación Acción Participante IAP:

Recordemos cómo mis colegas y yo, de países del tercer mundo, fuimos articulando desde los años de 1970 nuestro pensamiento y acción, combinando, como decíamos, corazón y cabeza para proponer técnicas y procedimientos que satisficieran nuestras angustias como ciudadanos y como científicos sociales ${ }^{10}$.

Los cuatro tomos (volúmenes) de Historia doble de la Costa son una muestra del retorno comprometido con los campesinos de su tierra natal, que había iniciado con la investigación de Campesinos de los Andes.

Definitivamente, los dos eventos que desmarcaron a Fals Borda de la monografía sociológica de Saucío en la dirección de la IAP fueron los respectivos congresos internacionales promovidos y organizados por él en Cartagena de Indias, en 1977 y 1997. El I Simposio mundial organizado por la Fundación para el Análisis de la Realidad Colombiana (Fundarco) tuvo como eje central el problema de la práctica en las ciencias

\footnotetext{
${ }^{8}$ Fals Borda, Orlando, Campesinos de los Andes, 5ta edición (Bogotá: Punta de Lanza, 1979), p. 309. Apéndice A.

9 Ibid., p. 307.

${ }^{10}$ Fals Borda, Orlando, La investigación en convergencias disciplinarias (Montreal, Canadá: Latin American Studies Association (lasa), septiembre de 2007), p. 2.
} 
sociales. Derivado de este evento, al que asistieron y participaron delegados de diecisiete países, Fals Borda sistematizó sus resultados en el texto "Por la praxis: cómo estudiar la realidad para transformarla"11.

Veinte años después del primer congreso de la incipiente IAP (Cartagena de Indias, abril de 1977), se realizó en esta ciudad del Caribe colombiano el 8o Congreso de Convergencia participativa, espacio y tiempo, del 31 de mayo al 5 de junio de 199712 .

De los múltiples resultados es pertinente destacar lo referente a una aproximación de la IAP redefinida como Investigación Participativa para diferenciarla de la Investigación Acción de la escuela psicologista y experimental de Kart Lewin de tradición clásica y positivista ${ }^{13}$.

En lo fundamental, a juicio de Fals Borda, en Cartagena creció el convencimiento sobre la bondad de la rebelión contra lo que Thomas Kuhn"14 definió como "ciencia normal" y "paradigma dominante", que se expresan en las instituciones establecidas. Son aquellas reglas de trabajo de empiristas lógicos, positivistas y funcionalistas derivadas del peso imitativo que sobre las disciplinas sociales han tenido las ciencias "duras" desde el siglo xix, incluidas las normas sobre objetividad y neutralidad.

Con base en lo desarrollado en Cartagena en 1997, en convergencias y disidencias, el coordinador Fals Borda propuso para discutir una definición inicial de la Investigación Participativa:

Es método de estudio y acción que va al paso con una filosofía altruista de la vida para obtener resultados útiles y confiables en el mejoramiento de situaciones colectivas, sobre todo para las clases populares. Reclama que el investigador o investigadora base sus observaciones en la convivencia con las comunidades de las que también obtiene conocimientos válidos. Es

\footnotetext{
11 Bogotá: Punta de Lanza, 1977.

12 Asistieron 1850 personas provenientes de 61 países y se presentaron 165 ponencias. Orlando

Fals Borda fue el coordinador general del evento. Compiló y editó el registro del Congreso con el aporte de Inmanuel Wallerstein, Agnes Heller, Fernando Henrique Cardoso, Manfred Max-Neef, Eduardo Galeano, Rajesh Tandon, Robert Flood, Alfredo Molano, Gustavo De Roux, Francine Fournier, Jaime Niño, Denis Goulet, ponentes y coordinadores de grupo de trabajo.

13 “Por las razones explicadas en la Parte III (Experiencias teórico-prácticas) de éste libro, se ha adoptado aquí el término "Investigación Participativa" (IP) como intercambiable con el de "Investigación-Acción Participativa" (IAP) que hemos usado desde los años 70", en Participación popular: retos del futuro. Compilación y análisis Orlando Fals Borda (Bogotá: ICFES-IEPRI-Colciencias, 1998), p. ix, pp. 182-183.

14 Ibid., pp. 172-173.
} 
inter o multidisciplinaria y aplicable en continuos que van de lo micro a lo macro de universos estudiados (de grupos a comunidades y sociedades grandes), pero siempre sin perder el compromiso existencial con la filosofía del cambio que la caracteriza ${ }^{15}$

La anterior noción de lo acumulado, a propósito de la IAP, elaborada porFals Borda contrasta con el método ecléctico identificado, seleccionado y definido en el apéndice A (primer anexo) de la investigación Campesinos de los Andes:

[El método de investigación] Consistía el primer problema práctico en determinar los medios y elementos para lograr los objetivos. La información directa y el contacto personal se consideraron indispensables. Así, como núcleo metodológico, se utilizaron formularios, diarios de campo y observación por participación. Tuve la esperanza de trasladarme a Saucío, donde pudiera vivir con una de las familias de la localidad; y después de cuatro meses de contactos y visitas, pude hacerlo. De esta manera tuve una buena oportunidad de estudiar y observar la vida entre los campesinos, prácticamente en todos sus diversos matices ${ }^{16}$.

Un resumen en versión libre de pautas indicadoras de las guías generales de los trabajos de la Investigación Participativa son las siguientes ${ }^{17}$ :

Resulta inocuo buscar o determinar leyes sociales generales en el campo social.

- El rigor investigativo no se juzga solo con mediciones cuantitativas, aunque estas puedan ser necesarias en la descripción y explicación de los resultados del trabajo.

- Por la fluidez natural de los fenómenos sociales, culturales, económicos y políticos, estos hay que verlos como procesos históricos sujetos a análisis hermenéuticos.

\footnotetext{
15 Ibid., p. 182.

16 "Estas notas personales acerca de la forma como el autor estableció contacto con el vecindario de Saucío son de interés, especialmente para los sociólogos que han de encontrarse en situaciones análogas en al América Latina. Estas notas también ilustran la manera como fue ejecutada la investigación sobre Saucío. Ambos procesos - el de construir puentes afectivos y sociales entre el investigador y la comunidad, y el aplicar un métodos adecuado de investigación - son sumamente pertinentes en relación con el texto que antecede". Fals Borda, Orlando, Campesinos de los Andes (Bogotá: Punta de Lanza, 1979), p. 309 y en la p. 307: El método y el

trabajo de campo.

17 Participación popular: retos del futuro. Compilación y análisis Orlando Fals Borda (Bogotá: ICFES-IEPRIColciencias, 1998). Ibid., pp. 192-196.
} 
- Todos los métodos y técnicas de las ciencias son legítimos de aplicar en la investigación participativa, siempre y cuando esto encaje dentro del marco de referencia.

- La empatía y la observación participante son las técnicas más apropiadas para investigar la naturaleza íntima o encubierta de las situaciones que interesan a la investigación participativa.

- Los criterios de validez para trabajos de la IAP dependen, no de pruebas de correlación interna de variables o ejercicios "objetivos" o cuantificables, sino del examen inductivo-deductivo de resultados determinables por la práctica, por el desarrollo empático de procesos sentidos en las mismas realidades, por juicios ponderados de grupos de referencia locales y por el sentido común.

- La evaluación de resultados no se ejecuta necesariamente al término dado o prefijado por el conocido ritmo de la reflexión-acción como si fuera un procedimiento bancario unilineal [...] La inspiración necesaria para continuar estas tareas es fractal [...].

- Los valores, metas y compromisos que animan a los investigadores participativos deben ser transparentes.

- No hay lugar para la experimentación a la manera de las ciencias naturales, que permitan la repetibilidad de fenómenos en contextos controlados.

- El romper la díada investigador-investigado para quebrar la asimetría y horizontalizar la relación, no significa el predominio intelectual de uno u otro polo.

- En determinadas circunstancias conviene distinguir entre la racionalidad investigativa y la racionalidad política, y no mezclarlas indiferentemente.

- La objetividad y la subjetividad pueden coincidir en el arte, en la imagen sensual y en la expresión literaria y estética del trabajo científico, lo que justifica la incursión creadora de la imaginación y la expresión en los trabajos de investigación participativa.

Con la anterior definición provisional de la IAP y las correspondientes guías metodológicas de los trabajos de campo, es necesario volver a leer, re-escribir y re-editar 
todo el proceso de investigación realizado en la vereda de Saucío en 1949-1953 y comparar la devolución sistemática de Campesinos de los Andes en la coyuntura actual colombiana de pos-conflicto y la construcción de la paz, como lo propuso e hizo Fals Borda en los prólogos y epílogos de El hombre y la tierra en Boyacá; Desarrollo histórico de una sociedad minifundista, en su tercera edición de 1979, La subversión en Colombia: El cambio social en la historia, con su cuarta edición actualizada en abril de 2008 cuatro meses antes su fallecimiento ${ }^{18}$.En el contexto no solo de la vereda de Saucío, sino desde todos los territorios de Colombia con el método de la praxis más sistematizado, el paradigma holístico alternativo en consolidación, la situación actual de la sociedad civil nacional, el sesquicentenario de la Universidad Nacional de Colombia y la Quinta Conferencia Internacional de Investigación Participativa PAR en el 2017 de ARNA, Participación y democratización del conocimiento: convergencias para la reconciliación. Se puede intentar establecer las concordancias y diferencias de la teoría, método y práctica falsbordiana, así como la posible utilidad y aplicación de la IAP para implementar las propuestas contenidas en el Acuerdo Final Pactado para el Fin del Conflicto y la Construcción de una Paz Estable y Duradera ${ }^{19}$, cuya ejecución pondría fin de manera definitiva a un conflicto armado de más de cincuenta años en Colombia, que coincide en sus inicios con la investigación realizada por Orlando Fals Borda al vecindario de Saucío a partir de 1949.

Al contrastar el análisis, las inferencias y recomendaciones contenidas en Campesinos de los Andes, en término de los quince capítulos y anexos y los contenidos

\footnotetext{
18 Por lo anterior, un grupo de jóvenes estudiantes iniciados en Sociología de la Universidad Nacional de Colombia, se dieron a la tarea - con la salida de campo incluida a la vereda de Saucío-de aproximarse a reescribir Campesinos de los Andes con una mirada retrospectiva de sesenta años. Los informes del ejercicio sobre los quince capítulos que integran los resultados de la investigación saucita arrojaron la siguiente aproximación, tomando en cuenta las tensiones estratégicas de la IAP (teoría-practica, sujetoobjeto y neutralidad-compromiso): olvido y resistencia, resiliencia rural, transición, vivencia, dilema de la tierra, la problemática de la investigación social, segunda parte de una historia, el hombre y la tierra alrededor de la modernidad, comunidad campesina, reconstrucción, cambio en los últimos cincuenta años, seis décadas del campesinado.

${ }^{19}$ Introducción: "El fin del conflicto supondrá la apertura de un nuevo capítulo de nuestra historia. Se trata de dar inicio a una fase de transición que contribuya a una mayor integración de nuestros territorios, una mayor inclusión social - en especial de quienes han vivido al margen del desarrollo y han padecido el conflicto- $-y$ a fortalecer nuestra democracia para que se despliegue en todo el territorio nacional y asegure que los conflictos sociales se tramiten por las vías institucionales, con plenas garantías para quienes participen en política". Firmado en La Habana, Cuba, el 27 de agosto de 2016. Mesa de conversaciones, Preámbulo, pp. 3-4. En la versión definitiva del Acuerdo Final, firmado el 12/24 de noviembre de 2016, p. 6 de $310 \mathrm{p}$.
} 
expresos en las 297 páginas del Acuerdo Final para la Terminación del Conflicto y la Construcción de una Paz Estable y Duradera ${ }^{20,82}$ se puede plantear hipotéticamente que este pacto-documento coincide, de manera general en buena parte, con las acciones de política formuladas por Fals Borda en el estudio sociológico de Saucío (reforma agraria, participación, víctimas y fin del conflicto), y abre el espacio para institucionalizar las reformas necesarias en el proceso de cambio socioeconómico y cultural de la población rural colombiana, en el marco del orden democrático que la carta política de 1991 establece y a la cual contribuyó a diseñar, redactar y a construir en su totalidad el constituyente Fals Borda, pero, especialmente, los Artículos 60, 64, 66 21, y los de ordenamiento territorial.

A pesar de las limitaciones teóricas, metodológicas y empíricas de los resultados del estudio de caso en referencia en el espacio y el tiempo, llama la atención la visión de Fals Borda sobre el problema agrario, más allá de lo propuesto en Campesino de los Andes y la manera como perfiló el campo de la sociología rural colombiana y latinoamericana, que guardada las proporciones, se aproxima a las consideraciones ${ }^{22}$ del punto uno (Hacia un Nuevo Campo Colombiano: Reforma Rural Integral) y complementariamente, del punto cuatro (Solución al Problema de las Drogas Ilícitas) ${ }^{23}$ del Acuerdo Final.

De todas maneras, el estudio pionero de La violencia en Colombia ${ }^{24}$, que publicó en 1962 y reeditó en 2005, así como el análisis de La subversión en Colombia ${ }^{25}$, en su versión del 2008, son referentes para estudiar el conflicto interno de los últimos cincuenta años

\footnotetext{
${ }^{20}$ Firmado en La Habana, Cuba, el 27 de agosto de 2016. Mesa de conversaciones, Preámbulo, p. 3. Suscrito el 24 de agosto de 2016. En la versión definitiva del Acuerdo Final, firmado el 12/24 de noviembre de 2016, p. 10 de 310 p.

${ }^{21}$ Artículo 64. "Es deber del Estado promover el acceso progresivo a la propiedad de la tierra de los trabajadores agrarios, en forma individual o asociativa, y a los servicios de educación, salud, vivienda, seguridad social, recreación, crédito, comunicaciones, comercialización de los productos, asistencia técnica y empresarial, con el fn de mejorar el ingreso y calidad de vida de los campesinos".

${ }^{22}$ República de Colombia (Gobierno nacional) y Farc-EP. Acuerdo final para la terminación del conflicto y la construcción de una paz estable y duradera (La Habana, Cuba: Mesa de conversaciones, 27 de agosto de 2016), pp. 8-297. En la versión definitiva del Acuerdo Final, firmado el 12/24 de noviembre de 2016, p. 98 de $310 \mathrm{p}$.

23 Ibid., pp. 88-297.

${ }^{24}$ Guzmán, Germán, Fals Borda Orlando y Umaña L. Eduardo. La violencia en Colombia, t. I y II (Bogotá: Editorial Iqueima, 1962).

${ }^{25}$ Fals Borda, Orlando, La subversión en Colombia. El cambio social en la historia, 4ta edición (Bogotá: FICACEPA, 2008)
} 
desde los territorios colombianos, lo cual, a su vez, está relacionado con lo planteado en el punto tres del Acuerdo Final: fin del conflicto ${ }^{26}$.

El origen de la información para adelantar la investigación sobre las causas de la confrontación bipartidista de hace más de medio siglo la aporta Fals Borda, confirmando su distancia del enfoque neopositivista con el que adelantó la investigación de Saucío:

En 1961 junto con Camilo (Torres) 2789 descubrimos la existencia del fondo de documentación de la Comisión Oficial de Estudio de las causas de la violencia, que había nombrado el presidente Alberto Lleras. El Secretario de esa comisión era Monseñor Germán Guzmán Campos, que tuvo la buena disposición de conservar esa documentación. Camilo me convenció de que fuéramos a visitar a Monseñor Germán Guzmán, que era entonces párroco del Líbano, Tolima [...] Allá vimos el archivo y lo convencimos que se viniera a trabajar a la Facultad de Sociología. [...] trabajamos juntos escribiendo el primer tomo sobre la violencia. Lo hicimos en secreto, nadie sabía que lo estábamos haciendo porque era muy delicado. Habíamos decidido decir las cosas con nombre propio, fechas y sitios. Teníamos toda la documentación necesaria a la mano. Al analizar ese trabajo, su intensidad, la naturaleza del conflicto, pues rompió en mi cabeza todo el esquema que había llevado del funcionalismo; no se puede explicar con el marco de referencia aprendido en las aulas de mis maestros. Escribí como conclusión de ese tomo mi primera expresión de alejamiento de ese modelo funcionalista, nosotros teníamos que asumir una posición mucho más clara, comprometida con las soluciones, y por eso el libro de la violencia termina con 27 o 30 recomendaciones al gobierno, a la sociedad colombiana, a la iglesia, y a la universidad, a todo el mundo, de cómo resolver el problema de la violencia. Son recomendaciones que si uno las lee todavía hoy eran muy lógicas, obvias, muy posibles; pero nunca fueron atendidas; fueron inspiradas precisamente en la sensación que teníamos de comprometernos con algo que sirviera a la sociedad. Una sociología comprometida con la transformación social28.90

Si bien es cierto que en el capítulo 5, "El hombre y la tierra", 6, "Evolución del poblamiento" y 7, "Características de la agricultura intensiva" de la segunda parte

\footnotetext{
${ }^{26}$ República de Colombia (Gobierno nacional) y Farc-EP. Acuerdo final para la terminación del conflicto y la construcción de una Paz estable y duradera (La Habana, Cuba: Mesa de conversaciones.

${ }^{27}$ Comparar la vigencia de la ponencia de Camilo Torres, "La violencia y los cambios socioculturales en las áreas rurales colombianas con su enfoque de sociología positivista" (Memorias del I Congreso Nacional de Sociología. Bogotá, marzo de 1961) con la Agenda del "Acuerdo de diálogo para la paz de Colombia entre el Gobierno Nacional y el ELN (marzo de 2016) con el siguiente orden de temas: 1. Participación de la sociedad; 2. Democracia para la paz; 3. Transformaciones para la paz; 4. Víctimas; 5 . Fin del conflicto; 6. Implementación de lo acordado,

inicio de la fase pública de los diálogos de paz entre el Gobierno y el ELN el 8 de febrero de 2017

${ }^{28}$ Cendales, L., Torres, F. y Torres, A., Uno siembra la semilla pero ella tiene su propia dinámica. Entrevista a Orlando Fals Borda. Pregunta: ¿En lo que fue haciendo y trabajando, qué pudo haber dado cauce a la IAP? Recuperado de Dimensión Educativa.org.co (2004).
} 
“Organización social" de Campesinos de los Andes, se estudia el problema de distribución, uso y tenencia de la tierra en la vereda de Saucío, es a partir del estudio La tierra y el hombre en Boyacá (1957) ${ }^{29}$, cuando se sientan las bases para estructurar una propuesta de reforma agraria no solo para la vereda de Saucío, sino para Colombia. Al respecto -en una entrevista-3092 Fals Borda aportó su testimonio del proceso que vivió para la investigación de las pequeñas propiedades boyacenses contiguas al municipio de Chocontá, cabecera de la provincia de Almeidas, en el departamento de Cundinamarca.

Yo sí vine acá cuatro meses para trabajar en la tesis del doctorado en Boyacá, por el problema del minifundio, la pobreza en Boyacá que nos llamó la atención tanto al profesor Smith como a mí. [...] Había que destacar la pobreza y el problema del campo como elementos esenciales para explicar la situación de atraso de Colombia y de la violencia actual. La violencia en Colombia como fenómeno político se inició en el campo, fue un enfrentamiento entre campesinos inducido desde arriba, impulsado por los políticos, por el propio presidente Ospina Pérez, o el ministro de gobierno José Antonio Montalvo, luego el presidente Laureano Gómez, horribles figuras de la historia colombiana, porque a ellos se les debe mucho de lo ocurrido después, porque la consigna que sembró Montalvo desde el Congreso era combatir "a sangre y fuego", esa fue la orden que le dio a los conservadores para combatir a los liberales. Menciono lo de Boyacá en el sentido de que el libro que luego saqué con mi tesis, tenía como subtítulo: "Bases para una reforma agraria" y que - todavía está pendiente ese problema - va al fondo de la cuestión nacional. El subtítulo me llevó al Ministerio de Agricultura. Una vez que salió el libro con propuestas sobre cómo hacer una reforma agraria en Colombia, primera vez que se planteaba el problema agrario en esa forma, aunque antes había los intentos de los socialistas como Gerardo Molina, Antonio García, Jorge Eliécer Gaitán, pero eran políticos. [...] Al salir el libro, inesperadamente, me llama Augusto Espinoza Valderrama, el Ministro de Agricultura del gobierno de Alberto Lleras, y me dice, bueno, leí su libro, me gusta mucho, venga para acá a ponerlo en práctica. La praxis.

Los planteamientos, análisis y propuestas de Fals Borda inventariados y relacionados anteriormente, se pueden estudiar a la luz de las consideraciones ${ }^{31}$, los

\footnotetext{
${ }^{29}$ Fals Borda, A Sociological Study of the Relationships between Man and Land in the Departament of Boyacá, disertación doctoral (Gainesville: Universidad de Florida, 1955)

${ }^{30}$ Cendales, L, Torres, F y Torres A, Uno siembra la semilla pero ella tiene su propia dinámica. Entrevista a Orlando Fals Borda. Pregunta: ¿Durante el tiempo del doctorado venía a Colombia? Recuperado de Dimensión Educativa (2004).

${ }^{31}$ República de Colombia (Gobierno nacional) y Farc-EP. Acuerdo final para la terminación del conflicto y la construcción de una paz estable y duradera (La Habana, Cuba: Mesa de conversaciones, 27 de agosto de 2016), pp. 8-29/297. En la versión definitiva del Acuerdo Final, firmado el 12/24 de noviembre de 2016, pp. $10-12$ de 310 p.
} 
principios $^{32}$, para establecer la vigencia de las investigaciones y las propuestas de un nuevo campo colombiano como se plantea en el punto uno del Acuerdo Final, en particular, las concordancias y diferencias, en relación con: ${ }^{33}$ primero, tierra: acceso y uso. Tierras improductivas. Formalización de la propiedad. Frontera agrícola y protección de zonas de reserva; segundo, Programas de Desarrollo con Enfoque Territorial (PDET) y, tercero, Planes Nacionales para la Reforma Rural Integral que incluyan infraestructura y adecuación de tierras, desarrollo social (salud, educación, vivienda, erradicación de la pobreza), y estímulos a la producción agropecuaria y a la economía solidaria y cooperativa con asistencia técnica, subsidios, crédito, generación de ingresos, mercadeo y formalización laboral ${ }^{34}$.

El punto dos del acuerdo "Participación política: apertura democrática para construir la paz", considera

[...] que la construcción y consolidación de la paz, en el marco del fin del conflicto, requiere de una ampliación democrática que permita que surjan nuevas fuerzas en el escenario político para enriquecer el debate y la deliberación alrededor de los grandes problemas nacionales y, de esa manera, fortalecer el pluralismo y, por tanto, la representación de las diferentes visiones e intereses de la sociedad, con las debidas garantías para la participación y la inclusión política ${ }^{35}$.

Desde sus épocas de juventud, Fals Borda fue descubriendo que la participación es fundamental para concretar los objetivos de los proyectos sociales, como lo aprendió de María Borda, líder de causas comunitarias en su natal Barranquilla.

No por azar Fals Borda es el iniciador de organizaciones comunales y cooperativas desde 1958, en las que puso a prueba la efectividad de participación de los campesinos en la construcción no solo de la escuela rural y el acueducto veredal con los saucitas, sino

\footnotetext{
32 Ibid.

33 Ibid.

${ }^{34}$ Ibid., pp. 11-29. En la versión definitiva del Acuerdo Final, firmado el 12/24 de noviembre de 2016, p. 14 de $310 \mathrm{p}$.

35 República de Colombia (Gobierno nacional) y Farc-EP. Acuerdo final para la terminación del conflicto y la construcción de una paz estable y duradera (La Habana, Cuba: Mesa de conversaciones, 27 de agosto de 2016), pp. 30-49/297. En la versión definitiva del Acuerdo Final, firmado el 12/24 de noviembre de 2016, pp. 35-55 de 310 p.
} 
también el trabajo con Camilo Torres ${ }^{36}$ en el barrio Tunjuelito de Bogotá a través de la Junta de Acción Comunal de la localidad.

A partir los años sesenta, el decano-profesor-extensionista, desde la Facultad de Sociología de la Universidad Nacional de Colombia, al confrontarse con la realidad social rural y urbana -en la que realizaban los trabajos de campo- fue descubriendo y construyendo colectivamente una herramienta de trabajo teórica-práctica que en esa época comenzó a ser llamada Investigación Telética con base en el concepto de telesis (telos signifca fnalidad) ${ }^{37}$ que privilegia la participación comprometida para transformar la realidad social con sentido altruista.

Las evidencias de la construcción, desarrollo, mejoramiento y afinamiento del método participativo del promotor Fals Borda están consignadas en los escritos de esta antología: La participación en la reforma agraria, la acción comunal, la participación popular, 38100 los problemas contemporáneos en la aplicación de la sociología al trabajar en la Investigación Acción Participativa, complementadas con las experiencias, testimonios y vivencias retornadas y compartidas en los congresos, simposios, seminarios, encuentros y demás eventos desde la realidad a transformar el pasado, presente y futuro.

Por su efecto transformador, la IAP en convergencias disciplinarias, se constituye en uno de los instrumentos más apropiado, necesario, justo y útil para la implementación del punto dos (Participación política: Apertura democrática para construir la paz) del Acuerdo Final ${ }^{39}$ en lo pertinente a garantizar: 1. Los derechos y garantías plenas para el ejercicio de la oposición política en general, y en particular, para los nuevos movimientos que surjan luego de la firma del Acuerdo Final, así como el acceso a medios de

\footnotetext{
${ }^{36}$ Contrastar con el punto uno "Participación de la sociedad", en Acuerdo de diálogo para la paz de Colombia entre el Gobierno nacional y el ELN (marzo de 2016).

${ }^{37}$ Fals Borda, Orlando, La subversión en Colombia. El cambio social en la historia, 4ta edición (Bogotá: FICA-CEPA, 2008), p. 11

38 Participación popular: retos del futuro compilación y análisis Orlando Fals Borda (Bogotá: ICFES/IEPRI/Colciencias, 1998). Presentación, V-IX. 24, parte iV "El futuro de las convergencias participativas", p. 21. Fals Borda, Orlando, La estrella polar del altruismo, pp. 251-255

${ }^{39}$ República de Colombia (Gobierno nacional) y Farc-EP. Acuerdo final para la terminación del conflicto y la construcción de una paz estable y duradera (La Habana, Cuba: Mesa de conversaciones, 27 de agosto de 2016), pp. 30-49/297.
} 
comunicación; 2. El acceso sin restricción a los mecanismos democráticos de participación ciudadana, incluidos los de participación directa en los diferentes niveles y diversos temas; y 3. La implementación de medidas efectivas para promover una mayor participación en la política nacional, regional y local, de todos los sectores, incluyendo la población más vulnerable, en igualdad de condiciones y con garantías de seguridad. ${ }^{40}$

En relación con el punto tres del Acuerdo Final, ${ }^{41} 103$ la resolución y superación del conflicto interno en Colombia, Fals Borda comenzó a identificar este aspecto en el estudio de Saucío (tesis de maestría de 1953), y luego, por la proximidad territorial, en su tesis doctoral de 1955: El hombre y la tierra en Boyacá, planteando como alternativa de solución a la confrontación en el campo, las "bases para una reforma agraria" a partir de las cuales, el Ministerio respectivo expidió la correspondiente Ley de Reforma Social Agraria (135 del 13 de diciembre de 1961) que él contribuyó a formular e implementar desde la Dirección de Agricultura con el apoyo de la Facultad de Sociología de la Universidad Nacional de Colombia.

Para el analista Fals Borda, el fin del conflicto colombiano está indisolublemente atado a superar las causas de la violencia endémica. En el lanzamiento de una nueva edición de La violencia en Colombia (2005), con una mirada retrospectiva y tomando como contexto la coyuntura en acumulación (1962-2005), afirmó que en el nuevo prólogo hay dos tesis centrales generales. La primera, interpreta a la llamada "violencia" como el resultado de una política destructiva del entorno y del tejido social, diseñada e impulsada conscientemente por una oligarquía que se ha querido perpetuar en el poder a toda costa, desatando el terror y la guerra. La segunda tesis sostiene que este proceso de patología social se viene repitiendo en ciclos más o menos acompasados, en los que se cambia el nombre del actor violento, directo o inspirador, por el del nuevo actor. ${ }^{42}$

En el caso de la confrontación armada en la región del Caribe colombiano, territorio considerado no azotado en principio con la fuerza de la violencia andina en la

\footnotetext{
${ }^{40}$ Ibid., pp. 32-35, pp. 36-43 y pp. 44-49/297.

${ }^{41}$ República de Colombia (Gobierno nacional) y Farc-EP. Acuerdo final para la terminación del conflicto y la construcción de una paz estable y duradera (La Habana, Cuba: Mesa de conversaciones, 27 de agosto de 2016), pp. 50-87/297. En la versión definitiva del Acuerdo Final, firmado el 12/24 de noviembre de 2016, pp. 56-97 de 310 p.

${ }^{42}$ Fals Borda, Orlando, La violencia en Colombia, Prólogo (Bogotá: Taurus, 28 de abril de 2005).
} 
década de los cincuenta hasta los sesentas, el caribeño Fals Borda demuestra cómo fue afectado progresivamente por la matiz (maríapalito) del conflicto a partir de la lucha por la tierra del campesinado costeño ${ }^{43}$ y la resistencia encarnada en la Asociación Nacional de Usuarios Campesinos ANUC línea Sincelejo.

Su comprensión del problema de violencia en su tierra de nacimiento la expresa considerando que el Caribe continental colombiano era reconocido como un remanso de paz para preguntarse: “qué había pasado en mi tierra desde 1948?”44 Supone que hubo un primer fatal descuido de la clase dominante por la suerte del campo, que era fuente de su riqueza y poder: "no sintieron la urgencia de la transformación por la justicia, dejando a las clases trabajadoras al arbitrio de la ley de la fuerza y de la explotación capitalista más salvaje". 45

Entonces, esta ley brutal se aplicó con cierta facilidad por agentes externos comprometidos con la violencia de interior del país, en la que también cayeron los dirigentes costeños. Con su vivencia señala que:

Sangre inocente y campesina fue cubriendo poco a poco veredas y playones, y fue subiendo hacia los Montes de María, por un lado, y por el otro por las ciénagas de mis primos, los hombres hicoteas de San Martín de Loba y Magangué, y por los rastros de mis abuelas chimilas de Mompox y de Pijiño. ${ }^{46}$

Manifesta que la mancha sangrienta hizo metástasis en el tejido social regional y se fue extendiendo más al norte sin que los dirigentes costeños actuaran para atajarla, hasta alcanzar los fabulosos paraísos del Cesar y del Ariguaní, y subió secando los 56 ríos de la Sierra Nevada de Santa Marta, hasta casi saturar con el terror la ancestral cultura del humor y del dejar hacer.

Sin embargo, aquella violencia extraña al terruño natal empezó a ser endógena. La geohistórica región Caribe está así dejando de ser costeña. “Estamos sucumbiendo a la

\footnotetext{
${ }^{43}$ Fals Borda, Orlando, Historia doble de la Costa, t. III Resistencia en el San Jorge (1984), vol. 4 (Bogotá: Carlos Valencia Editores, 1986), p. 34B, pp. 136B-164B.

${ }^{44}$ Fals Borda, Orlando, En defensa de la costeñidad y la paz Caribe: mi gran frustración (Barranquilla: Periódico El Heraldo, 28 de marzo de 2004) y en Hacia el socialismo raizal y otros escritos (Bogotá: Ediciones Desde abajo y CEPA, agosto de 2007) y "Me queda la angustia de la continuidad", Revista Foro n. 65 (Bogotá, octubre de 2008), pp. 5-10.

${ }^{45} \mathrm{Ibid}$.

46 Ibid.
} 
violencia foránea y a la delincuencia resultante". El asesinato del sociólogo Alfredo Correa De Andrei, entre otras, impulsó a Fals Borda a escribir, en un especial estado de angustia, “De poco han servido 'Mensajes' musicales, libros, revistas, sermones y discursos. Tampoco leyes, decretos y bravatas de gobernantes". ${ }^{47}$

Lapidariamente termina el mensaje haciendo un llamado angustioso:

Por eso, mis paisanos, colegas y amigos, esta es mi mayor frustración como sociólogo y como ser humano. Pasé casi toda mi vida en guerras múltiples, a veces deformadas, por el narcotráfico o sufriendo sus trágicas consecuencias, tratando de entenderlas y explicarlas, combatiendo el belicismo con ideas, propuestas y algo de malicia indígena. Pero ya no tengo tiempo, en mi vejez, de seguir campaneando sobre la Violencia o por la Segunda República, apenas esbozarlas, como es mi actual preocupación. ${ }^{48}$

Invocando el ethos transformador de Saucío de Campesinos de los Andes y del Caribe colombiano, para el fin del conflicto y la construcción de una paz estable y duradera, Fals Borda podría coincidir con uno de los considerandos del punto tres -fin del conflicto-y con algunas medidas pactadas en los puntos dos y tres del Acuerdo Final:

El Gobierno nacional reafirma su compromiso con lo acordado en los puntos 3.4 y 3.6 del punto 3 Fin del Conflicto, entre los que se encuentra la creación de un nuevo Sistema Integral de Seguridad para el Ejercicio de la Política, en los términos acordados en el punto 2 Participación Política, como parte de una concepción moderna, cualitativamente nueva de la seguridad que, en el marco del fin del conflicto, se funda en el respeto de la dignidad humana, en la promoción y respeto de los derechos humanos y en la defensa de los valores democráticos, en particular en la protección de los derechos y libertades de quienes ejercen la política, especialmente de quienes luego de la terminación de la confrontación armada se transformen en movimiento político y que, por tanto, deben ser reconocidos y tratados como tales. ${ }^{49}$

\footnotetext{
47 Fals Borda, Orlando, En defensa de la costeñidad y la paz Caribe: mi gran frustración (Barranquilla: Periódico El Heraldo, 28 de marzo de 2004) y en Hacia el socialismo raizal y otros escritos (Bogotá: Ediciones Desde abajo y CEPA, agosto de 2007) y "Me queda la angustia de la continuidad" Revista Foro N. 65 (Bogotá, octubre de 2008).

${ }^{48}$ Ibid.

${ }^{49}$ República de Colombia (Gobierno nacional) y Farc-EP. Acuerdo final para la terminación del conflicto y la construcción de una paz estable y duradera (La Habana, Cuba: Mesa de conversaciones, 27 de agosto de 2016), pp. 50, 87 y 297. En la versión definitiva del Acuerdo Final, firmado el 12/24 de noviembre de 2016, pp. 124-191 de 310 p.
} 
El punto cinco del Acuerdo trata sobre las "víctimas"112 del conflicto y las identifica de la siguiente manera:

La terminación de la confrontación armada signifcará, en primer lugar, el fin del enorme sufrimiento que ha causado el conflicto. Son millones los colombianos y colombianas víctimas de desplazamiento forzado, cientos de miles los muertos, decenas de miles los desaparecidos de toda índole, sin olvidar el amplio número de poblaciones que han sido afectadas de una u otra manera a lo largo y ancho del territorio, incluyendo mujeres, niños, niñas y adolescentes, comunidades campesinas, indígenas, afrocolombianas, negras, palenqueras, raizales y rom, partidos políticos, movimientos sociales y sindicales, gremios económicos, entre otros. No queremos que haya una víctima más en Colombia. ${ }^{50}$

Fals Borda siguiendo los pasos de los marxistas peruanos Mariátegui y Arguedas, recomendó volver los ojos, respetar y reaprender de cuatro pueblos que han conformado la esencia de la nación colombiana, que son víctimas del conflicto armado y a los que hay que reparar. Son ellos: los indígenas, los negros de palenques, los artesanos y campesinos antiseñoriales y los patriarcas colonos de la frontera agrícola. De estos se deben recuperar valores sociales fundantes, de presencia reconstructiva y universal, respectivamente así: la solidaridad, la libertad, la dignidad y la autonomía. ${ }^{51}$

Es reiterativo en la necesidad de recuperar las raíces de los pueblos originarios y sus valores dominantes para tener una mayor comprensión de las realidades y problemas territoriales actuales y para los propósitos de la indispensable reconstrucción social que demanda la paz colombiana.

Concretamente plantea cuatro retos a la IAP en un escenario de posconflicto: ${ }^{52}$ primero, la construcción de un paradigma alterno, estudiando los grupos originarios o fundantes regionales de víctimas, destacando sus valores de reconciliación y solidaridad humana. Segundo, entender y combinar, en el contexto regional, las complejidades de las sociedades en posconflicto: lo oral, lo particular, lo local. Tercero, los métodos para

\footnotetext{
50 Ibid.

${ }^{51}$ Fals Borda, Orlando, La investigación en convergencias disciplinarias (Montreal, Canadá: Latin American Studies Association lasa, septiembre de 2007), p. 7.

52 Fals Borda, Orlando, Situación contemporánea de la IAP y vertientes afines. Ponencia presentada para el 1er Encuentro Internacional de Investigadores en Acción, Universidad Nacional Experimental Rafael María Baralt, Cabimas Estado Zulia, Venezuela, 22 de junio de 2006, p. 114.
} 
resistir la homogeneización de la globalización para defender las identidades y la vida como naciones y pueblos autónomos.

El educador popular Fals Borda considera que en estos desafíos las universidades y otras instituciones deberían estar prestas a apoyar esta monumental tarea y ajustarse a sus necesidades.

A propósito del sesquicentario de la Universidad Nacional de Colombia, su propia experiencia y retorno definitivo a la tierra ${ }^{53}$ del alma mater, Fals Borda llama la atención de las implicaciones y retos de la IAP para las instituciones de educación superior. ${ }^{54} 117$ Reconoce que en estas ha habido un proceso de cooptación participativa, ya que son para el 2006 por lo menos 2500 universidades de 61 países y 32 escuelas regionales, en las que se enseña o practica la Investigación Acción Participativa. Pero advierte que los intentos de llevar a la práctica estos trabajos con profesores y estudiantes se ha difcultado por la inflexibilidad de los pénsum, los ritmos —en semestres o años - de la enseñanza y la exigencia de tesis individuales para optar títulos. ${ }^{55}$

Como se analizó en Cartagena (1997),,56 estos trabajos son de mediano y largo plazo y requieren de una continuidad mínima, con persistencia y compromiso personal o de equipos, por lo menos por parte de profesores y alumnos que mantengan el pulso de los trabajos más allá de cualquier semestralización.

Consideró el docente Fals Borda

No siempre se combinan estas condiciones en las instituciones para construir el vínculo universidad-sociedad de manera participativa. Pesan mucho el elitismo y el negocio. Aun así, es fácil ver que asumir estas tareas con decisión llevaría a transformaciones fundamentales en las estructuras y funciones de las universidades: algo que muchos jóvenes estudiantes han

\footnotetext{
53 Las cenizas de Orlando Fals Borda fueron depositadas el 19 de junio de 2014 en el mausoleo frente a la Capilla de la Sede Bogotá de la Universidad Nacional de Colombia.

54 Participación popular: retos del futuro. Compilación y análisis Orlando Fals Borda (Bogotá: CFES/IEPRI/Colciencias, 1998), p. 210.

55 Ibid., pp. 210-211.

56 Ibid.
} 
venido sintiendo y exigiendo, para que aquellas se sintonicen mejor con la vida extrauniversitaria y con la realidad que nos condiciona a todos. ${ }^{57}$

En términos de retos a la educación superior, la investigación sociológica de caso en la vereda de Saucío, su recuperación crítica en la monografía de Campesinos de los Andes y la devolución sistemática de la Historia doble de la Costa, son puntos de referencia y una oportunidad para redefinir las misiones de las instituciones universitarias en un escenario de construcción social de una paz desde los territorios colombianos en el marco del Acuerdo Final para la terminación del conflicto y su implementación con el apoyo de la IAP.

Si bien es cierto que en la encrucijada actual de Colombia, se reconoce la coherencia de vida y obra, la vigencia del legado del sentipensante- - especialmente a las nuevas generaciones-, sus reiterados llamados al diálogo constructivo, a la tolerancia, a la diferencia y al compromiso; el maestro mayor murió con la frustración y la angustia por la continuidad de los esfuerzos para encontrar los caminos sostenibles para la reconciliación nacional y superar el conflicto interno. ${ }^{58}$

La publicación de Campesinos de los Andes y otros escritos antológicos por la Universidad Nacional de Colombia, en sus 150 años de fundada, ayuda a disminuir la angustia con la cual falleció Orlando Fals Borda hace ocho años, y avanzar en la construcción de un paradigma holístico alterno a través de la investigación acción participante en convergencias disciplinarias para que, al trascender los enfoques positivistas, funcionales y mecánicos, aporte respuesta a las críticas situaciones de colombianas y colombianos.

La circulación, lectura y apropiación crítica en clave de la IAP del texto antológico de Orlando Fals Borda, debe contribuir a cambiar el aparente ethos de la "pasividad" de los campesinos de Saucío, los Andes y el Caribe, en un ethos transformador de las condiciones socioeconómicas, culturales y políticas de los grupos más vulnerables de Colombia en la perspectiva de posconflicto y la construcción de la paz.

57 Participación popular: retos del futuro Compilación y análisis Orlando Fals Borda (Bogotá: ICFES/IEPRI/Colciencias, 1998), p. 211.

58 Ibid. 


\section{Referencias Bibliográficas}

CATAÑO, Gonzalo. (2008). Orlando Fals Borda, sociólogo del compromiso. Revista de Economía Institucional 10, No 19 pp. 79-98.

CASTILLO CÁRDENAS, Gonzalo y BENAVIDES ISAY, Pérez. (2010). La influencia religiosa en la conciencia social de Orlando Fals Borda. Barranquilla: Corporación Universitaria Reformada.

CENDALES, Lola., TORRES, F. y TORRES, Alfonso. (2004). Uno siembra la semilla pero ella tiene su propia dinámica. Entrevista a Orlando Fals Borda. Recuperado de Dimensión Educativa.org.co (2004).

FALS BORDA, Orlando. (1955) A Sociological Study of the Relationships between Man and Land in the Departament of Boyac. Disertación doctoral. Gainesville: Universidad de Florida.

FALS BORDA, Orlando. (1977) Por la praxis: cómo estudiar la realidad para transformarla. Bogotá: Punta de Lanza.

FALS BORDA, Orlando. (1979) Fals Borda, Orlando, Campesinos de los Andes, 5ta edición, Bogotá: Punta de Lanza, p. 309. Apéndice A.

FALS BORDA, Orlando. (2004). En defensa de la costeñidad y la paz Caribe: mi gran frustración. Barranquilla: Periódico El Heraldo.

FALS BORDA, Orlando. (2005). La violencia en Colombia, Bogotá: Taurus.

FALS BORDA, Orlando. (2006). Situación contemporánea de la IAP y vertientes afines. Ponencia presentada para el 1er Encuentro Internacional de Investigadores en Acción, Universidad Nacional Experimental Rafael María Baralt, Cabimas Estado Zulia, Venezuela.

FALS BORDA, Orlando. (2007) Hacia el socialismo raizal y otros escritos, Bogotá: Ediciones Desde abajo y CEPA, agosto.

FALS BORDA, Orlando. (2007). La investigación en convergencias disciplinarias. Montreal, Canadá: Latin American Studies Association (lasa), septiembre.

FALS BORDA, Orlando. (2008) "Me queda la angustia de la continuidad", Revista Foro n. ${ }^{\circ}$ 65: Bogotá, octubre, pp. 5-10.

FALS BORDA, Orlando. (2008). La subversión en Colombia. El cambio social en la historia, 4ta edición. Bogotá: FICA-CEPA. 
IEPRI. (1998) Participación popular: retos del futuro. Compilación y análisis Orlando Fals Borda. Bogotá: ICFES-IEPRI-Colciencias.

GUZMÁN, Germán, FALS BORDA Orlando y UMAÑA L. Eduardo. (1962). La violencia en Colombia, T. I y II. Bogotá: Editorial Iqueima.

REPÚBLICA DE COLOMBIA (Gobierno nacional) y FARC-EP. (2016) Acuerdo final para la terminación del conflicto y la construcción de una paz estable y duradera (La Habana, Cuba: Mesa de conversaciones, 27 de agosto de 2016. Versión definitiva del Acuerdo Final, firmado el 12/24 de noviembre de 2016. 